$z$ a distance $\pm \delta z$. One of these examples retains a center of symmetry, but the other does not; they result if the longitudinal or transverse phonon at the zone boundary point in the 100 direction goes unstable. It is worth noting that there is a peak in superconducting transition temperature near the $\mathrm{Tl}$ concentration $(\sim 30 \%)$ at which this transition occurs near $0^{\circ} \mathrm{K}$. This reinforces the notion that it is second-order.

We would like to acknowledge valuable conversations with W. L. McMillan, B. W. Batterman, R. C. Miller, B. T. Matthias, and E. J. Ryder.

\footnotetext{
${ }^{1}$ B. W. Batterman and C. S. Barrett, Phys. Rev. Letters 13 , 390 (1964).

${ }^{2} \mathrm{~J}$. P. Maita and E. J. Ryder, private communication.

${ }^{3}$ L. Guttman, Trans. AIME 188, 1472 (1950).

${ }^{4}$ L. D. Landau and E. M. Lifshitz, Statistical Physics (Addison-Wesley Publishing Company, Inc., Reading, Massachusetts, 1958), Chap. XIV. This theory is at present considered to be incorrect in detail at the pre-
}

cise transition point, but the basic symmetry considerations do not seem to depend on the small singular terms which destroy the validity of the expansion (1) when $T \rightarrow T_{c}, \eta \rightarrow 0$. In particular, we believe our physical argument for $\eta=(c / a)-1$ to be obviously valid with a greater degree of rigor than the expansion.

${ }^{5}$ These statements can be supported by a group theoretical demonstration. This and a complete classification of the various possible symmetry transformations will be published shortly.

${ }^{6}$ See, for example, W. Kohn and Vachaspati, Phys. Rev. $\underline{83}, 462$ (1951).

${ }^{7}$ A. F. Devonshire, Phil. Mag. $\underline{60}, 1040$ (1949); $\underline{62}$, 1065 (1951). This effect is the result of the coupling of internal symmetry to strain, which often adds a negative effective fourth-order term to Eq. (1). This strain effect can also be discussed more fully ${ }^{5}$

${ }^{8} \mathrm{P}$. W. Anderson, Proceedings of the All-Union Conference on the Physics of Dielectrics of the Academy of Sciences of the U.S.S.R., December 1958, p. 290. W. Cochran, Phys. Rev. Letters $\underline{3}, 412$ (1959); Advan. Phys. 9387 (1960).

${ }^{9}$ J. W. Stout and L. Guttman, Phys. Rev. $\underline{88}, 703$, 713 (1952).

\title{
PHOTOEMISSIVE DETERMINATION OF BARRIER SHAPE IN TUNNEL JUNCTIONS
}

\author{
A. Braunstein, M. Braunstein, and G. S. Picus \\ Hughes Research Laboratories, Malibu, California \\ and \\ C. A. Mead \\ California Institute of Technology, Pasadena, California \\ (Received 6 January 1965)
}

Tunnel junctions have been characterized in terms of three parameters, the barrier heights $\varphi_{1}$ and $\varphi_{2}$ and the width $S$, which generally are determined by a fit of experimental current-voltage characteristic curves with theory. In metal-semiconductor systems barrier heights have been determined independently of other parameters from measurement of the spectral dependence of photoresponse. ${ }^{1,2} \mathrm{We}$ wish to report the first results of the application of this technique to the measurement of the barrier heights in $\mathrm{Al}-\mathrm{Al}_{2} \mathrm{O}_{3}-\mathrm{Al}$ and $\mathrm{Al}-\mathrm{Al}_{2} \mathrm{O}_{3}$ $\mathrm{Au}$ tunnel junctions where the $\mathrm{Al}_{2} \mathrm{O}_{3}$ thickness is in the range of 20 to $40 \AA$.

The sample structures were fabricated by evaporating aluminum strips onto glass microscope-slide substrates at a vacuum of $10^{-6}$ Torr. The surface of these strips was oxidized in an oxygen glow discharge,$^{3}$ and the structure was completed by evaporation of a thin semitransparent counterelectrode of aluminum or gold. All 15 samples on a given slide showed uniformity of $J-V$ characteristics and photoemissive properties. The data reported here are for a typical sample.

$\mathrm{Al}-\mathrm{Al}_{2} \mathrm{O}_{3}-\mathrm{Al}$ tunnel junctions are observed to display asymmetric $J-V$ characteristics, from which it is inferred that an intrinsic field exists in the $\mathrm{Al}_{2} \mathrm{O}_{3}$. Therefore, the experimental photoresponse must be analyzed on the basis of a trapezoidal barrier model such as is shown in the inset in Fig. 1(a). Two basic types of photoprocesses are possible. For photon energies $h \nu<\varphi_{1}$ the excited electrons can only tunnel through the barrier. For higher photon energies electrons can also be injected into the conduction band of the oxide over the barriers. In the latter case we assume that hot electrons lose their energy to the lattice in a distance small compared to the thickness of the oxide. ${ }^{4}$ Hence electrons traveling against the electric field do not contribute to the pho- 
tocurrent. Since $\mathrm{Al}_{2} \mathrm{O}_{3}$ is transparent to radiation at all photon energies used in these experiments, and the hot-electron mean free path in the metal electrodes is short compared to the optical absorption distance, we further assume that both electrodes are approximately equally illuminated. Therefore, for Al$\mathrm{Al}_{2} \mathrm{O}_{3}-\mathrm{Al}$ samples the density of photoexcited electrons can be taken to be the same on both sides of the barrier. We now consider the net photocurrent expected in the $\mathrm{Al}-\mathrm{Al}_{2} \mathrm{O}_{3}-\mathrm{Al}$ system for various applied voltages and incident photon energies on the basis of the above assumptions.

With no voltage applied to the sample, and for photon energies less than $\varphi_{1}$, only equal opposed photon-induced tunnel currents can flow, and there should be no net photocurrent. For photon energies between $\varphi_{1}$ and $\varphi_{2}$, electrons from electrode 1 must traverse the conduction band of the insulator against the intrinsic field in order to tunnel through the barrier, contributing less to the photocurrent than electrons from electrode 2. Therefore, there is a net photon-induced tunnel current in the $J_{2}$ direction. Finally, for photon energies greater than $\varphi_{2}$, hot electrons originating in electrode 1 cannot contribute significantly to the current because of their small mean free path in the oxide and the presence of the intrinsic field. Electrons originating in electrode 2 are aided by the intrinsic field, and so a $J_{2}$ emission current over the barrier is observed. If the square root of the photocurrent in this region is plotted as a function of photon energy, a straight line is obtained as shown in Fig. 1(a).$^{1,2}$ The barrier height $\varphi_{2}$ obtained from the intercept of this line on the energy axis is $1.77 \mathrm{eV}$.

When a voltage $V$ is applied such that electrode 1 is positive, a photoresponse curve similar to that at zero applied voltage is obtained. The low-energy tail becomes more pronounced since now the photoexcited electrons in the two electrodes differ in energy by $e V$ and those with higher energy (electrode 2) have a higher tunneling probability. In addition, the barrier height $\varphi_{2}$ should be reduced as the voltage is increased due to the Schottky high-field effect. As the bias is increased from 0 to 0.35 volt in the $J_{2}$ direction, $\varphi_{2}$ is observed to decrease from 1.77 to $1.72 \mathrm{eV}$.

Application of a voltage such that electrode 2 is positive produces a more complicated situation. For voltages less than $\Delta \varphi=\varphi_{2}-\varphi_{1}$ and for photon energies less than $\varphi_{1}$, the response from electrode 1 predominates. When the incident photon energy is greater than $\varphi_{2}$, photoemissive currents over the barrier occur in both directions. The $J_{1}$ current is attenuated due to the intrinsic field so the $J_{2}$ current must eventually predominate and produce a reversal in the sign of the photocurrent. The exact location of the crossover depends on a small difference in two large currents, and therefore its energy is difficult to predict. If the applied voltage in this direction is equal to $\Delta \varphi$, the intrinsic field is removed and the two tunneling responses should again be observed, but there should be no response from electrons excited over the barrier. Figure 1(b) illustrates a situation for which this condition is very nearly met. The presence of the crossover indicated that the applied voltage is less than $\Delta \varphi$. Finally, if $V$ is greater than $\Delta \varphi$, the response is all due to electrode 1 and the curves

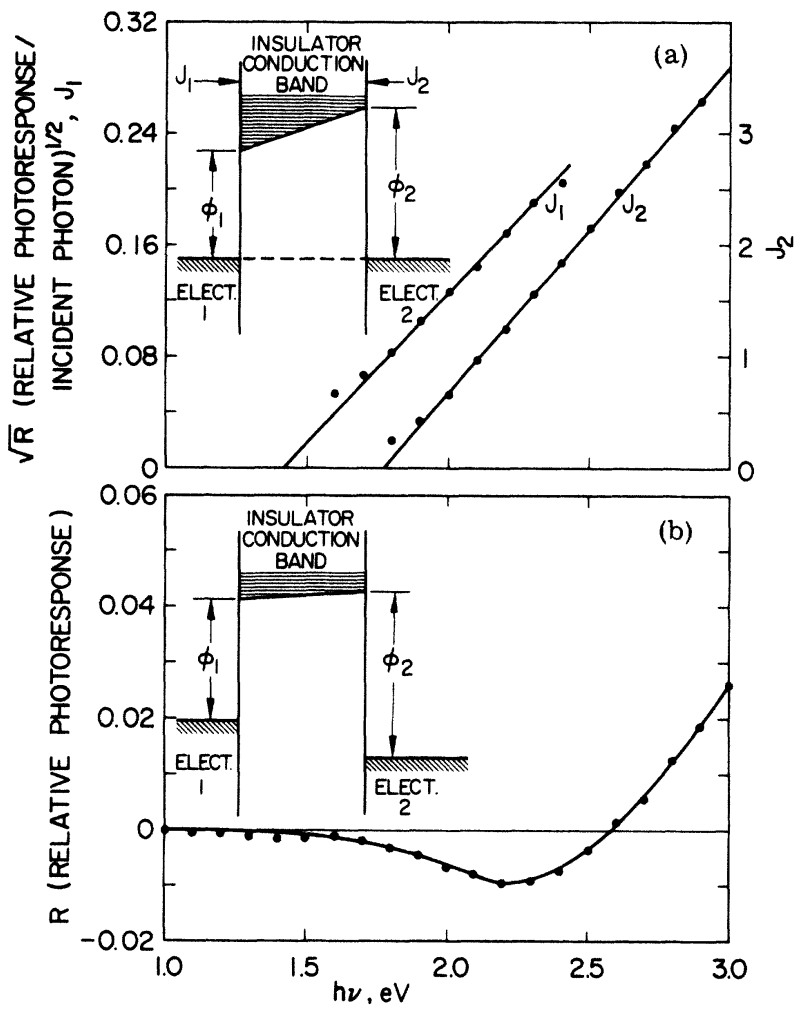

FIG. 1. (a) Square root of response per incident photon versus photon energy. $J_{2}$ curve (right-hand scale) is for zero bias; $J_{1}$ curve (left-hand scale) is for a bias of $0.53 \mathrm{~V}$ with electrode 2 positive. (b) Relative photoresponse versus photon energy for a bias of $0.4 \mathrm{~V}$ with electrode 2 positive. Positive response is defined as flow of electrons from electrode 2 to electrode 1 . This is the case of almost zero internal field in the $\mathrm{Al}_{2} \mathrm{O}_{3}$, as illustrated in the inset. 
are similar to the $J_{2}$ curve of Fig. 1(a). In this case, however, one measures the barrier height $\varphi_{1}$ as shown by the curve labeled $J_{1}$ in Fig. 1(a), and the value obtained is $1.42 \mathrm{eV}$.

The difference of the experimental values of $\varphi_{1}$ and $\varphi_{2}$ does not give the correct result for $\Delta \varphi$ because they include the Schottky-effect lowering of the barrier. Even the value of $\varphi_{2}$ measured at zero bias must be corrected for lowering by the intrinsic field. Using the theory of this effect in the form presented by Simmons, ${ }^{5}$ a pair of simultaneous equations were derived involving differences of experimental barrier heights and the unknowns $\Delta \varphi$ and $K S\left(K=\mathrm{Al}_{2} \mathrm{O}_{3}\right.$ dielectric constant, $S=$ barrier thickness in $\AA)$. These were solved graphically to obtain $\Delta \varphi=0.43 \mathrm{eV}$ and $K S=272 \AA$, and corrected barrier heights of $1.49 \mathrm{eV}$ and $1.92 \mathrm{eV}$ for $\varphi_{1}$ and $\varphi_{2}$, respectively. This value for $\Delta \varphi$ is consistent with the disappearance of the photoresponse crossover [Fig. 1(b)] between $0.4 \mathrm{~V}$ and $0.53 \mathrm{~V}$ in the $J_{1}$ direction. The assumption of a strong electron-lattice interaction in the $\mathrm{Al}_{2} \mathrm{O}_{3}$ (giving a short mean free path) suggests that the low-frequency dielectric constant should be used in the Schottky effect equations. If the above value of $K S=272 \AA$ is combined with the value of $K / S=0.233 / \AA$ obtained from $100 \mathrm{kc} / \mathrm{sec}$ capacitance measurements, we obtain $K=8.0$ consistent with the value of the dielectric constant of bulk $\mathrm{Al}_{2} \mathrm{O}_{3}$, and $S$ $=34.0 \AA$.

For samples in which the counterelectrode is gold, similar results are obtained, with two exceptions. First, the intrinsic field is much larger due to the work-function difference between aluminum and gold. This produces larger photon-assisted tunneling responses in the $J_{2}$ direction due to the greater effect of the intrinsic field. Second, gold is observed to be much less efficient than aluminum in the generation of photoelectrons in the energy range of interest. This results in a crossover in the response at low photon energies even with no applied voltage, due to the larger number of photoexcited electrons in the aluminum (electrode 1 ). The observed intrinsic field in $\mathrm{Al}-\mathrm{Al}_{2} \mathrm{O}_{3}-\mathrm{Au}$ structures is approximately 0.9 volt, in reasonable agreement with the work-function difference.

${ }^{1}$ C. R. Crowell, W. G. Spitzer, L. E. Howarth, and L. E. LaBate, Phys. Rev. 127, 2006 (1962).

${ }^{2}$ C. A. Mead and W. G. Spitzer, Appl. Phys. Letters 2 , 74 (1963).

${ }^{3}$ J. L. Miles and P. H. Smith, J. Electrochem. Soc. $\underline{110}, 1240$ (1963).

${ }^{4}$ R. E. Collins and L. W. Davies, Solid-State Electron. 7,445 (1964).

${ }^{5}$ J. G. Simmons, J. Appl. Phys. 35, 2472 (1964).

\section{DISLOCATION STRUCTURE IN ULTRASONICALLY IRRADIATED ALUMINUM}

K. H. Westmacott and B. Langenecker

Research Department, U. S. Naval Ordnance Test Station, China Lake, California

(Received 4 January 1965)

Intense ultrasonic radiation may produce pronounced hardening in metal crystals. ${ }^{1}$ For example, the critical shear stress in $99.999 \%$ pure aluminum was found to be increased by a factor of three to four after a $0.1-\mathrm{sec}$ pulsed irradiation of $25 \mathrm{~W} / \mathrm{cm}^{2}$ ultrasound at $20 \mathrm{kc} /$ sec. $^{2}$

Langenecker ${ }^{1}$ interpreted this acoustic hardening (also referred to as acoustic radiation damage) as a result of a reduction in the mean free path of dislocations. This reduction was assumed to be caused by the continuous formation of obstacles such as vacancy rings during acoustically activated dislocation oscillations (amplitudes of oscillations $\geqslant 1 \mu$ ). No direct evidence for this hypothesis was given, nor were dislocation and sound theories adequate in describing the phenomena of interactions of high-intensity ultrasonic stress waves with dislocations.

We have continued investigations on mechanisms of acoustic radiation damage, and through the application of transmission electron microscopy a more detailed picture is now emerging.

We used 2.3-mm diameter and 50- $\mathrm{mm}$ long wire specimens of $99.999 \%$ aluminum (obtained from United Mineral and Chemical Company), which prior to irradiation were annealed at $500^{\circ} \mathrm{C}$ for $5 \mathrm{~min}$, and oven-cooled. Ir radiation with ultrasound was accomplished by screwing 